\title{
Large Dynamic Range Simulations of Galaxies Hosting Supermassive Black Holes
}

\author{
Robyn Levine ${ }^{1}$ \\ ${ }^{1}$ Canadian Institute for Theoretical Astrophysics, \\ 60 St. George St., Toronto, ON, M6G 1A6, Canada \\ email: levine@cita.utoronto.ca
}

\begin{abstract}
The co-evolution of supermassive black holes (SMBHs) and their host galaxies is a rich problem, spanning a large-dynamic range and depending on many physical processes. Simulating the transport of gas and angular momentum from super-galactic scales all the way down to the outer edge of the black hole's accretion disk requires sophisticated numerical techniques with extensive treatment of baryonic physics. We use a hydrodynamic adaptive mesh refinement simulation to follow the growth and evolution of a typical disk galaxy hosting an SMBH, in a cosmological context (covering a dynamical range of 10 million!). We have adopted a piecemeal approach, focusing our attention on the gas dynamics in the central few hundred parsecs of the simulated galaxy (with boundary conditions provided by the larger cosmological simulation), and beginning with a simplified picture (no mergers or feedback). In this scenario, we find that the circumnuclear disk remains marginally stable against catastrophic fragmentation, allowing stochastic fueling of gas into the vicinity of the SMBH. I will discuss the successes and the limitations of these simulations, and their future direction.
\end{abstract}

Keywords. galaxies: evolution, galaxies: high-redshift, galaxies: nuclei

\section{Introduction}

Observations suggest that most galaxies host massive compact objects in their centers, likely to be supermassive black holes (SMBHs) with masses ranging from $10^{6} \mathrm{M}_{\odot}$ to $10^{9} \mathrm{M}_{\odot}$ (e.g., Kormendy \& Richstone 1995; Magorrian et al. 1998). The ubiquity of $\mathrm{SMBH}$ at the centers of galaxies includes our own galaxy, where the use of adaptive optics has constrained the mass of the Milky Way's black hole to be $\approx 4.5 \times 10^{6} \mathrm{M}_{\odot}$ (Ghez et al. 2008).

Observations of galaxies hosting SMBHs indicate a relationship between black hole mass and various properties of the host galaxies, such as the spheroid mass (Magorrian et al. 1998) and the velocity dispersion of stars in the bulge (Ferrarese \& Merritt 2000; Gebhardt et al. 2000; Tremaine et al. 2002). In order for such correlations to arise, there must be some processes linking the growth of SMBHs (objects with a sphere of influence of order tens of parsecs) to the evolution of their host galaxies (governed by interactions on much larger, cosmological scales).

Active galactic nuclei (AGNs) undoubtedly are an important piece of the puzzle of galaxy and SMBH co-evolution. They output an enormous amount of energy into the host galaxy through both radiative and mechanical feedback, which can potentially influence galaxy evolution on scales ranging from that of the interstellar medium (ISM) to the intracluster medium. Since AGN are driven by accretion onto SMBHs, it is essential to understand black hole fueling. On galactic scales, fueling is determined by both the secular evolution of the host galaxy and by outside influences from the galaxy's environment (such as mergers with neighboring galaxies). Fueling may occur continuously over the course of some large-scale dynamical instability in the galaxy, or it may be an 
intermittent process, dependent entirely on the dynamics on small scales. Intermittent accretion episodes consisting of in-falling clouds of gas with randomly oriented angular momentum vectors may contribute to the spin-down of SMBHs, thus lowering their radiative efficiency and allowing them to grow faster (e.g., King \& Pringle 2007; King, Pringle, \& Hofmann 2008). Modeling the many physical processes relevant for the growth of SMBHs and their host galaxies is a challenging numerical feat, spanning a large range of scales.

In an attempt to meet the numerical challenge,we use cosmological adaptive mesh refinement (AMR) simulations with a large dynamic range to study the transport of gas and angular momentum through the circumnuclear disk of a SMBH host galaxy over time. Here we present some results of these simulations, which are presented in greater detail in our previous work (Levine et al. 2008; Levine 2008; Levine, Gnedin, \& Hamilton 2010).

\section{Simulations of Black Hole-Galaxy Co-Evolution}

Different Approaches. There are a range of numerical tools that are individually suited for addressing different pieces of the problem of SMBH-Galaxy co-evolution. Cosmological simulations follow the evolution of large scale structure and are well suited for following sequences of events occuring slowly over cosmic time (such as following merger histories, etc.). Often cosmological simulations implement sub-grid models to describe processes occurring on scales smaller than the resolution of the simulations (e.g. gas cooling, star formation, and feedback processes). Large-scale simulations often cannot follow the circumnuclear regions of galaxies with high enough resolution to describe the accretion onto the SMBH in detail, and must make approximations using the properties of the galaxies on scales that are resolved. A common practice is to estimate the Bondi accretion rate to describe the growth of the black hole (Bondi 1952). Such simulations can be sued to study the effects of merger-driven fueling and AGN feedback on SMBH growth and galaxy morphology and demographics (Sijacki et al. 2007; Di Matteo et al. 2008; Colberg \& Di Matteo 2008; Croft et al. 2009).

Small-scale simulations focus their attention on individual galaxies or even smaller scales inside them (e.g. accretion disks, star formation, or the ISM). High-resolution simulations of subgalactic scale disks, like those found is SMBH host galaxies, have found the development of a turbulent, multi-phase ISM (e.g., Wada 2001; Escala 2007; Wada \& Norman 2007). If the galactic disk is also self-gravitating, then this implies that accretion onto a central SMBH in such an environment is not well modeled by the Bondi accretion rate, as is often approximated in larger-scale simulations.

Adaptive Mesh Refinement and the Zoom-In Technique. Following both the complex baryonic physics of the ISM and star formation, and the cosmological evolution of a galaxy simultaneously, requires the use of adaptive simulation techniques. We address the issue of SMBH fueling using state-of-the-art cosmological simulations which are ideally suited to meet the numerical challenges presented above. The simulations use the AMR technique to self-consistently model the gas dynamics in a single galaxy at high resolution (subparsec resolution in the center of the galaxy). A large dynamic range ( $>10$ million), achievable with the AMR technique, allows us to bridge cosmological scales to scales relevant for molecular cloud formation (the birthplace of stars) and AGN fueling. It is a complex task to implement mergers, feedback, and secular evolution in large, cosmological simulations all at once. Our approach is to split the problem into pieces to be addressed one at a time, ultimately building a more realistic simulation. After studying the physics in this basic model galaxy, we can begin to include physical processes that are directly 
relevant to the problem of SMBH growth in the context of galaxy evolution, such as AGN feedback.

We have performed simulations using the Adaptive Refinement Tree (ART) code (Kravtsov, Klypin, \& Khokhlov 1997; Kravtsov 1999; Kravtsov, Klypin, \& Hoffman). The code follows gas hydrodynamics on an adaptive mesh, and includes dark matter and stellar particles (with stars forming at an observationally motivated rate), as well as gas cooling by heavy elements and dust, using rates tabulated from the CLOUDY code (Ferland et al. 1998). Additionally, radiative transfer and feedback and enrichment from stars are included in the pre-zoomed, cosmological portion of the simulation.

Beginning with a cosmological simulation with a maximum resolution of $\approx 50 \mathrm{pc}$ proper at $z=4$, the resolution is slowly increased one refinement level at a time in a region centered on a typical disk galaxy (which will evolve into a galaxy about the size of the Milky Way at $z=0$ ). The simulated galaxy reaches a quasi-stationary state on each level before the simulation refines to the next level. The final maximum resolution is $\approx 0.03 \mathrm{pc}$, corresponding to 20 levels of refinement. After reaching the maximum resolution, a fraction of the gas in the center of the galaxy is replaced with a black hole particle of equal mass and momentum. For simplicity, the mass of the black hole particle does not change over the course of any of our current zoom-in simulations. After the introduction of the black hole particle, the simulation continues to evolve with the maximum resolution for several hundred thousand years, allowing us to follow the evolution of the circumnuclear region of the galaxy at high-resolution. The zoom-in technique is also applied at different redshifts of the cosmological simulation, and for different black hole masses.

\section{Results}

Mass Accretion Rate. As the simulation evolves at high resolution, the circumnuclear disk becomes globally unstable, driving super-sonic turbulence. The turbulence supports the gas disk against catastrophic fragmentation into star forming clumps, maintaining marginal stability in the disk. The complex dynamical state of the circumnuclear disk results in transient structures forming on a range of scales. This behavior causes the gas mass interior to any radius within the disk to vary significantly on short timescales. Figure 1 shows the amplitude of fluctuations in the gas mass interior to four different radii throughout the circumnuclear region (different panels) over time for three different redshift simulations (different lines). The Figure gives an indication of the turbulent nature of the gas in the circumnuclear disk of the galaxy, and suggests that a characteristic accretion rate onto the $\mathrm{SMBH}$ is not straightforwardly determined by any one individual snapshot of the circumnuclear region. Instead, the accretion rate fluctuates randomly on short time scales because of the complicated dynamics of the circumnuclear disk. This result is qualitatively similar to the findings of Hopkins \& Quataert (2010), who use a different simulation technique (involving increasingly smaller re-simulations of the central disk) to reach high resolution in their SPH galaxy simulations.

Angular Momentum. Several authors have discussed the likelihood that gas which accretes onto a black hole can influence its spin (e.g., King \& Pringle 2007; Volonteri, Sikora, \& Lasota 2007; Berti \& Volonteri 2008; King et al. 2008). Whether accreting gas increases or decreases the spin of the black hole (ultimately determining its accretion efficiency and potentially the mode of feedback it produces) depends on the mass and angular momentum of the accreting material. Our simulations model gas on parsec scales, enabling us to resolve the direction of the angular momentum vector of gas (measuring position and velocity relative to that of the black hole particle) as it evolves over time. 


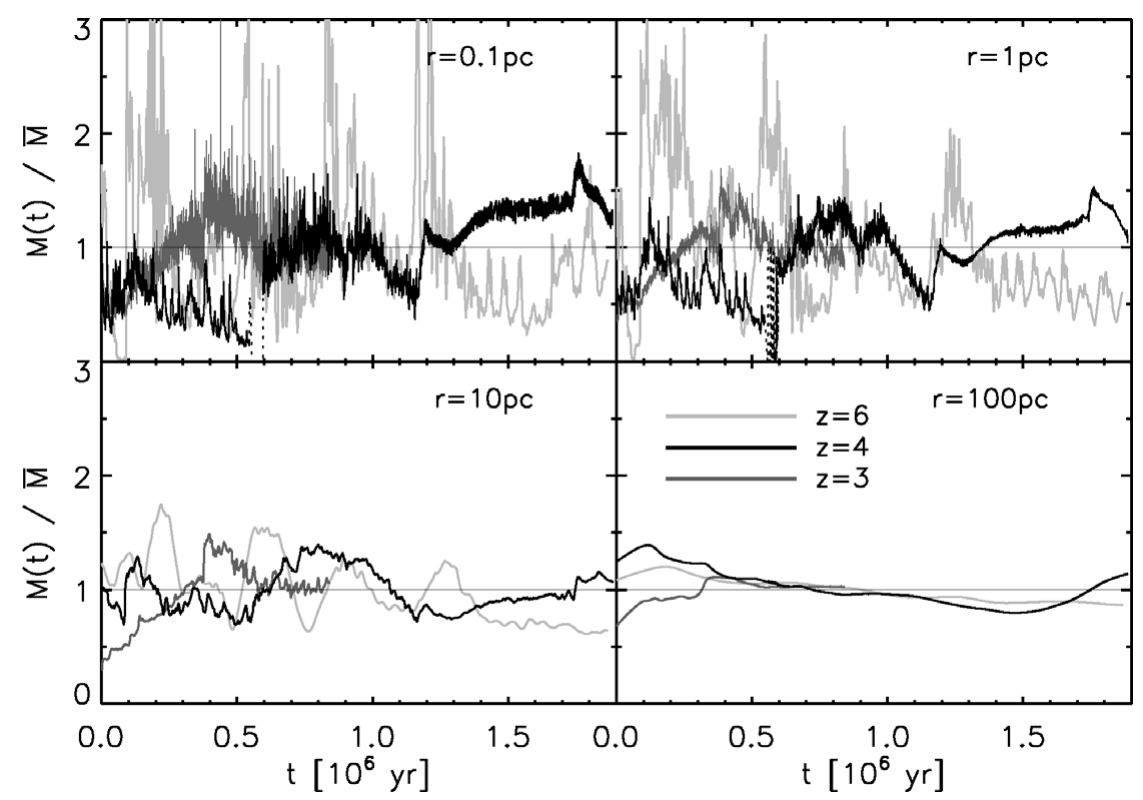

Figure 1. Amplitude of gas mass fluctuations at redshifts 3, 4, and 6 for 4 different radii (measured with respect to the location of the black hole particle). The thin solid line shows $M=\bar{M}$ for comparison.

Figure 2 shows a map projection of the direction of the normalized angular momentum vector, $\hat{L} \mathbf{L} / L$, as it evolves at 1 and $100 \mathrm{pc}$ from the black hole particle (for our $z=4$ simulation). The maps are oriented so that the angular momentum vector of the disk on kiloparsec scales lies in the center. The disk is slightly warped in the circumnuclear region, so that the axis of the disk is oriented at an angle to the large-scale disk.

At 1 pc (bottom), the direction of the angular momentum vector starts out at approximately the same orientation as kiloparsec scales, but shows slightly more scatter. The map shows two sudden changes in the direction of the angular momentum vector by 100 degrees each. The first incident, at $t \sim 0.55 \mathrm{Myr}$, occurs as a clump of gas with mass comparable to that of the black hole falls into the center. The scatter in the measurement of the angular momentum vector near this time (visible at $100 \mathrm{pc}$ as well) corresponds to a temporary displacement of the black hole particle as the clump reaches the center. The second incident, at $t \sim 1.2 \mathrm{Myr}$, is the result of gravitational interaction with a massive clump of gas which develops at $>100 \mathrm{pc}$ and continues to move toward the center of the disk at late times. Similar angular momentum flips occur over the course of several other runs with varying black hole masses.

At 100 pc (top), the angular momentum vector slowly changes direction, showing little scatter over the course of the simulation. The slow change in direction at $100 \mathrm{pc}$ may correspond to the increased warping of the disc as the simulation progresses. The behavior of the angular momentum vector, particularly on small scales in the simulations, consistently shows that the gas ultimately delivered to the SMBH may have varying angular momentum, which can change as massive clumps of gas develop and move through the disk. 


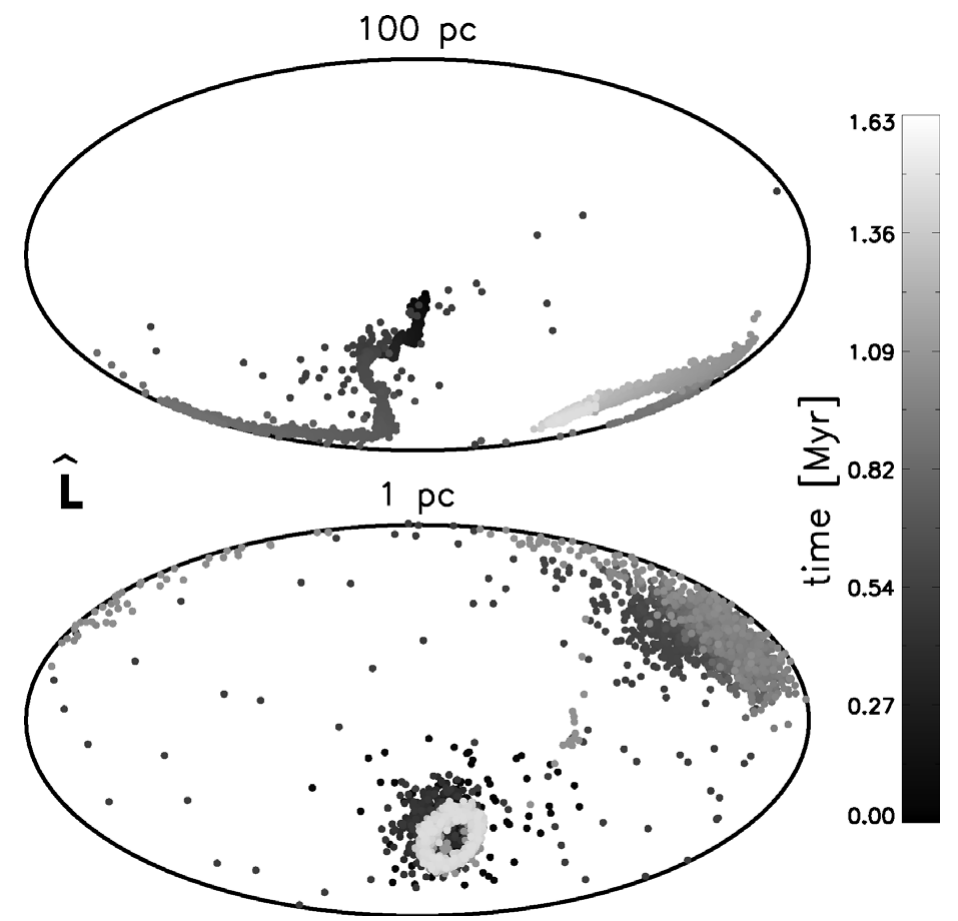

Figure 2. Map showing the direction of the normalized angular momentum vector, $\mathbf{L} / L$, as it changes over time in the $z=4$ simulation. Grey-scale corresponds to the elapsed time since the introduction of the black hole particle. The maps are aligned so that the mean rotation axis of the galactic disk on a scale of $1 \mathrm{kpc}$ sits in the center.

\section{Conclusions}

With a high-resolution cosmological simulation of a galaxy hosting an SMBH, we have modeled a simplified picture of accretion through a circumnuclear disk (without feedback from the black hole and in the absence of any major mergers). We found the development of a turbulent, self-gravitating, and globally unstable disk in the central kiloparsec. Accretion through this disk is stochastic, and not well modeled by the usual prescriptions employed in coarser resolution simulations. As clumps develop in the disk, they can potentially influence the angular momentum of the accretion disk on small scales, and subsequently the spin of the black hole.

A detailed analysis of accretion and angular momentum of gas requires accurate modeling of an array of physical processes that are important for galaxy evolution. In particular, resolving star forming regions and accurately modeling ISM physics can potentially change the dynamics of the circumnuclear disk. In new and improved simulations with the ART code, we plan to add additional physics important for studying black hole and galaxy co-evolution (such as feedback from black holes and black hole mergers) in order to better understand accretion onto black holes and the influence they have on their environment.

\section{References}

Berti, E. \& Volonteri, M. 2008, ApJ, 684, 822

Bondi, H. 1952, MNRAS, 112, 195 
Colberg, J. \& Di Matteo, T. 2008, MNRAS, 387, 1163

Croft, R. A. C., Di Matteo, T., Springel, V., \& Hernquist, L. 2009, MNRAS, 400, 43

Di Matteo, T., Colberg, J., Springel, V., Hernquist, L., \& Sijacki, D. 2008, ApJ, 676, 33

Escala, A. 2007, ApJ, 671, 1264

Ferland et al. 1998, PASP, 110, 761

Ferrarese, L. \& Merritt, D. 2000, ApJL, 539, L9

Gebhardt, K. et al. 2000, ApJL, 539, L13

Ghez, A. M. et al. 2008, ApJ, 689, 1044

Hopkins, P. F. \& Quataert, E. 2010, MNRAS, pub. online 21 July, 2010

King, A. R., Pringle, J. E., \& Hofmann, J. A. 2008, MNRAS, 385, 1621

King, A. R. \& Pringle, J. E. 2007, MNRAS, 377, L25

Kormendy, J. \& Richstone, D. 1995, ARAA, 33, 581

Kravtsov, A. V., Klypin, A. A., \& Khokhlov, A. M. 1997, ApJS, 111, 73

Kravtsov, A. V. 1999, Ph.D. Thesis, New Mexico State University

Kravtsov, A. V., Klypin, A. A., \& Hoffman, Y. 2002, ApJ, 571, 563

Levine, R., Gnedin, N. Y., Hamilton, A. J. S., \& Kravtsov, A. V. 2008, ApJ, 678, 154

Levine, R. 2008, Simulating the growth of a disk galaxy and its supermassive black hole in a cosmological context, Ph.D. thesis, University of Colorado at Boulder

Levine, R., Gnedin, N. Y., \& Hamilton, A. J. S. 2010, ApJ, 716, 1386

Magorrian, J. et al. 1998, AJ, 115, 2285

Sijacki, D., Springel, V., Di Matteo, T., \& Hernquist, L. 2007, MNRAS, 380, 877

Tremaine, S. et al. 2002, ApJ, 574, 740

Volonteri, M., Sikora, M., \& Lasota, J. P. 2007, ApJ, 667, 704

Wada, K. 2001, ApJL, 559, L41

Wada, K. \& Norman, C. A. 2001, ApJ, 547, 172

\section{Discussion}

VASIL: Is the Zeldovich approximation era over once the solutions to burger equation form shocks?

LEVINE: Yes, because once shocks develop, the temperature of the gas rises, introducting additional pressure so that the motion of gas particles is no longer described by the Zeldovich approximation. The Zeldovich approximation era precedes the formation of proto-galaxies, and we apply the zoom-in technique well after this point.

BRUN: Angular momentum transport depends strongly on the turbulence level. How confident are you that your simulations are "converged" in a hydrodynamical sense? What is the Reynolds number of your simulation?

LEVINE: We are convinced that the level of turbulence we measure at our resolution limit (and the implied turbulent viscosity) is consistent with the dynamics of the gas that we see. We've done a couple different things- measuring the turbulent viscosity by modeling the evolution of passive scalars, varying our resolution and the refinement technique, and all of these things produce consistent results. However, even though our results are self-consistent, there could be additional important effects that we fail to model because they're beneath our resolution limit. Our Reynolds number is probably enormous because the circumnuclear disk is very centrally concentrated (so that the velocities are quite high), but it's possible that fragmentation on small scales would lead to star formation, which would change the structure of the disk (and subsequently the dynamics). 
Combes: Your gaseous accretion disk appears very clumpy and unstable around the black hole; however it should be dominated by the mass of the black hole. Shouldn't it be only slightly self-gravitating?

LEVINE: Actually, the mass of the disk is enormous compared to the mass of the black hole, which we arbitrarily assigned to be $3 \times 10^{7} \mathrm{M}_{\odot}$ (in our fiducial simulation). So the disk is highly self-gravitating and the black hole doesn't influence the dynamics at all. The disk in these simulations has a very high gas fraction, but in future simulations, done with a newer version of the code, the gas fraction of the galaxy will be lower, so the black hole could potentially play a bigger role.

NTORMousi: How do you compare your black hole accretion model to Bondi-Hoyle accretion model usually employed in cosmological simulations?

LEVINE: Since the gas disk is so massive, it is self-gravitating and really doesn't resemble the Bondi regime at all. We attempted to estimate the Bondi accretion rate by using modified prescriptions accounting for turbulence and vorticity, but the rates even they suggest are enormous and don't at all resemble the behavior we observe. One of our goals though is to understand how the high-resolution simulations map onto lower resolution, cosmological simulations, where a Bondi prescription seems to give reasonable results (on average). That's a work in progress. 